\title{
Multilinguales
}

\section{Analyse discursive du forum de discussion algérien algérie-dz.com}

Discursive analysis of the algerian discussion forum algerie-dz.com

$$
\text { تحليل }
$$

:algerie-dz.com

\section{Yahia Abdeldjebar Atmane}

\section{(2) OpenEdition}

\section{Journals}

Édition électronique

URL : http://journals.openedition.org/multilinguales/736

DOI : 10.4000/multilinguales.736

ISSN : 2335-1853

Éditeur

Université Abderrahmane Mira - Bejaia

Référence électronique

Yahia Abdeldjebar Atmane, "Analyse discursive du forum de discussion algérien algérie-dz.com », Multilinguales [En ligne], 7 | 2016, mis en ligne le 02 juillet 2018, consulté le 17 septembre 2019. URL: http://journals.openedition.org/multilinguales/736 ; DOI : 10.4000/multilinguales.736

Ce document a été généré automatiquement le 17 septembre 2019.

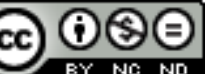

Multilinguales est mise à disposition selon les termes de la Licence Creative Commons Attribution Pas d'Utilisation Commerciale - Pas de Modification 4.0 International 


\title{
Analyse discursive du forum de discussion algérien algérie-dz.com
}

Discursive analysis of the algerian discussion forum algerie-dz.com

\author{
تحليل \\ خطابي

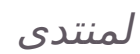

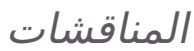 \\ الجزائري \\ :algerie-dz.com
}

Yahia Abdeldjebar Atmane

1 L'apparition des pratiques scripturales en ligne offre aux spécialistes de l'analyse du discours de nouvelles opportunités d'investigation. Plusieurs disciplines se focalisent, en effet, sur l'étude de ce nouveau langage: la linguistique, la didactique, l'informatique, la psychologie, la sociologie, etc.

2 Nous avons choisi d'analyser le forum algérien de discussion <ALGÉRIE-DZ.COM> pour le confronter à la thèse qui nous intéresse ici, à savoir celle de la double dimension d'un forum telle que prônée par Hassan Atifi et Michel Marcoccia (1998 - 2006): une dimension standard, internationale, et une dimension nationale, plus spécifique.

3 Nous tenterons de montrer que ce forum est une combinaison du style numérique au "niveau global» («concision, ménagement des faces, utilisation des smileys", Hassan Atifi et Michel Marcoccia, 2006) et du style numérique «au niveau local» (« variation culturelle ", id.) à travers notamment les alternances codiques. 
Pour cette investigation, nous avons eu recours à certains concepts de la sociolinguistique, de l'analyse conversationnelle et de l'approche interactionnelle.

\section{Un bref état de l'art}

5 La recherche dans le domaine du langage électronique a été initiée par Jacques Anis dans les années 80/901. Il a commencé par se pencher sur les messages du Minitel. A ce sujet, François Mangenot (2012) souligne, dans un article consacré en partie aux innovations de ce chercheur, qu'

à l'époque, Anis et Lebrave (1986) ou Anis (1987) désignaient la communication minitélique par les expressions, qui pouvaient presque à l'époque passer pour des oxymores, «textes interactifs " ou "écrit interactif », expressions justifiées par le fait que l'utilisateur pouvait non seulement recevoir des messages mais aussi en envoyer; apparaissait alors un «nouveau type de communication écrite quasi immédiate (Anis et Lebrave, 1986, p. 126).

Il retient que, «finalement, "les interactions entre les différents utilisateurs du système" seront considérées comme " l'aspect le plus intéressant [...] dans la mesure où le Minitel a créé [...] une nouvelle forme de communication, qui apparaît comme une forme hybride" entre l'écrit et l'oral (op. cit., p. 127)».

7 «Ces formes hybrides, entre l'écrit et l'oral» apparaissent sous diverses dénominations, répertoriées par R. Panckhurst (2009) qui justifie son propre choix pour celle de « communications médiées »:

Nous avons proposé une expression néologique pour le français « communication médiée par ordinateur » (« médiée » par opposition à «médiatisée » (Panckhurst, 1997), ou plus précisément dans un contexte linguistique « discours électronique médié » (Panckhurst, 1999, 2006, 2007). D'autres chercheurs proposent des variantes terminologiques pour décrire les phénomènes d'analyse linguistico-informatique de ces types de discours. Entre autres: « communication médiatisée par ordinateur 》 (Marcoccia, 2000), «communication électronique scripturale» (Anis, 2003), «nouvelles formes de communication écrite» (Guimier de Neef \& Véronis, 2004), «communication électronique» (Anis, de Fornel, Fraenkel, 2004) et, pour l'anglais : «Netspeak» (Crystal, 2001), « computermediated communication (Herring, 1996).

Les termes retenus dans un contexte (journalistique) plus vaste (comprenant également la publication électronique) incluent notamment: "cyberl@ngue ", « cyberlangage » (Dejond, 2002, 2006). (2009: 34-35)

Malgré la diversité de leurs appellations, ces nouveaux genres se caractérisent tous par l'usage de nouveaux codes comme les codes iconiques des smileys, le langage hybride entre l'oral et l'écrit et des ponctuants spécifiques.

Les recherches menées sur les écrits numériques s'interrogent, entre autres, sur la nature de ce nouveau genre de discours, pour déterminer s'il relève de l'écrit ou de l'oral.

Pour Anis (2000), ce type n'est à proprement parler ni écrit ni oral :

ce type de communication particulier, que l'on peut dénommer conversation écrite, se distingue à la fois de la situation canonique de la communication face à face par la parole et de la situation canonique de communication en différé par l'écrit (Anis, $2000: 57)$.

De « conversation écrite » d'Anis, Maingueneau a forgé la notion de « conversécriture » (2014) : 
[conversécriture] qui, à divers titres, subvertit la distinction traditionnelle entre échange immédiat (conversation) et différé (écrit), comme cela se voit au niveau des ressources linguistiques : ces types d'énoncés "écrits" possèdent de nombreux traits caractéristiques de l'oralité conversationnelle. (2014:187)

11 Notre article sera consacré à l'étude d'un type particulier de « communication médiée (ou «médiatisée ») par ordinateur ", ou encore de "conversécriture »: le forum de discussion au sens de Marcoccia : " un dispositif de communication médiatisé par ordinateur asynchrone, permettant à des internautes d'échanger des messages au sujet d'un thème particulier » (2004:26).

Les messages postés ne s'adressent pas à une seule personne mais à l'ensemble des internautes qui peuvent intervenir :

Dans un forum de discussion, il est impossible de sélectionner un destinataire.

Toute intervention est "publique", lisible par tous les participants au forum, même

si elle se présente comme la réaction à une intervention, initiative particulière.

L'aparté est impossible : le polylogue est la forme habituelle du forum et le multi-

adressage en est la norme. (Marcoccia, $1998: 17$ )

13 Le forum ainsi défini, sous forme de polylogue avec multi-dressage, constitue un observatoire des pratiques, des attitudes et des représentations des internautes.

Cette spécificité du forum nous intéresse particulièrement pour appréhender le forum conversationnel algérien.

En effet, à travers cette étude nous tenterons de cerner les modalités d'organisation des échanges discursifs spécifiques aux Algériens.

La question du caractère culturel des forums a déjà été traitée. Comme signalé plus haut, en 2006, Hassan Atifi et Michel Marcoccia, par exemple, se sont appliqués à démontrer que : "s'il exist[ait] un style des écrits numériques à un niveau global (concision, ménagement des faces, utilisation des smileys, etc.), il existe aussi certainement un style des écrits numériques à un niveau local : français, marocain, nord-américain, japonais, etc. ".

Mais déjà en 2003, Hassan Atifi avait publié, dans cette perspective, des travaux sur les forums de discussion marocains. Dans un article intitulé «La variation culturelle dans la communication en ligne: analyse ethnographique des forums de discussion marocains ", il écrit :

Dans cet article, nous étudions les usages que des internautes marocains font des normes du "savoir communiquer" dans leurs pratiques langagières authentiques: des forums de discussion en ligne. Nous poursuivons un double objectif. D'une part, nous voulons voir comment ces internautes marocains s'approprient des règles planétaires ou globales et mettre en évidence des particularismes culturels. Ces manifestations locales peuvent être en décalage, en rupture, voire en contradiction avec les normes communicatives normatives de la nétiquette.

L'auteur conclut que «la communication dans les forums marocains semble plus marquée par l'écart, la différence, et l'hétérogénéité que par la standardisation $»^{2}$.

Pour appréhender les spécificités culturelles qui surdéterminent les forums de discussion français et marocains, Atifi H. et Marcoccia M. proposent quatre pistes : « le style communicatif en ligne comme reflet du style " national », " la nétiquette comme facteur de standardisation culturelle », " le rapport entre style communicatif et sujet traité », et "l'importance des communautés virtuelles et des diasporas en ligne » (2006).

17 La « nétiquette » est ainsi définie par Marcoccia $(2000: 316)^{3}$. : 
Pour préserver la "qualité" des échanges, les acteurs centraux du réseau (...) ont élaboré un code de savoir-communiquer afin de susciter une attitude d'autorégulation chez les interactants. Ce code est appelé la nétiquette : l'étiquette $\mathrm{du}$ net. La nétiquette se présente comme un système normatif axiologisé. C'est un ensemble de règles fixes déterminant "ce qu'il faut faire".

Marcoccia extrait les règles de base de la nétiquette de trois textes présentés comme fondateurs de la «nétiquette de référence » (2000, op.cit. : 316-319): V. Shea : Netiquette (1994), S. Hambridge : Netiquette Guidelines (1995), et A. Rinaldi : The Net: User Guidelines and Netiquette (1996). Ces règles de base de la nétiquette sont :

- les «règles de politesse »: règles externes dictées par l'environnement social, règles internes au forum, et les commentaires métacommunicatifs entre internautes;

- la « règle de coopération » : concept inspiré du " principe de coopération » de Grice (1979) : « soyez pertinent », « clair et bref $»^{4}$;

- le « contenu des messages » : pas de message commercial, raciste, sexiste, trop personnel ;

- l'«identification des internautes": «la nétiquette incite les internautes à apporter dans leur message des informations manquantes et, surtout à ne pas tirer profit du dispositif technique pour endosser une fausse identité ».

19 Pour notre part, nous étudierons un forum de discussion algérien à travers sa mise en œuvre des règles de la nétiquette, c'est-à-dire des règles standard, globales du genre, et dans sa spécificité locale à travers notamment l'alternance codique.

Ce langage hybride, tel qu'il se pratique sur les forums, présente plusieurs intérêts. En premier lieu, il peut renseigner sur les profils culturels des internautes. En second lieu, il permet de maintenir le contact entre les forumeurs dans les situations d'interaction. L'alternance codique permet, en effet, de tisser des relations de connivence, de familiarité entre les internautes : "l'alternance codique permet d'établir une complicité, une intimité et peut générer un plaisir très grand, accessible aux jeunes bilingues » (Caubet, 2001 : 124).

21 Elle favorise également l'intervention au niveau du message lui-même. Selon Louise Dabène (1994 : 95-96),

on peut, en effet, observer que l'alternance codique offre au sujet bilingue toute une série de possibilités (....), permettant par exemple, de signaler le changement d'interlocuteur, l'insertion d'une citation, le passage à un autre registre de discours, l'effet d'insistance ou d'emphase, la recherche d'un mot plus précis, le commentaire sur ce qui vient d'être dit, etc.

22 Cette complicité entre partenaires de l'échange est l'effet d'une interaction qui peut être comprise comme :

L'influence réciproque que les partenaires exercent sur leurs actions respectives lorsqu'ils sont en présence physique immédiate les uns des autres par une interaction, on entend l'ensemble de l'interaction qui se produit en une occasion quelconque quand les membres d'un ensemble donné se trouvent en présence continue les uns des autres; le terme de "rencontre" pourrait convenir aussi. (Goffman, 1973 : 23)

Les échanges discursifs entre les intervenants, ou leur interaction/rencontre, supposent donc:

la présence de deux ou plusieurs participants ;

- l'influence mutuelle pendant l'échange (la perspective dialogique est mise en avant avec le respect de l'ordre hiérarchique) ;

- la présence physique des participants à l'échange ; 
- le déroulement de l'échange discursif hic et nunc.

enons enfin que, bien qu'écrits, les messages médiés par ordinateur sont plus proches de l'oral; les traits de l'oralité se manifestant par le recours à l'alternance codique, l'emploi de tournures idiomatiques ou idiosyncrasiques et les procédés du style parlé.

\section{Présentation du corpus}

Notre corpus est extrait du forum algérien de discussion Forum Algérie ${ }^{5}$. Ce site offre un large éventail de rubriques très diversifiées, allant de l'assistance informatique au club de rencontre, en passant par la musique, la cuisine, la santé, l'actualité, le divertissement, etc.

Le forum est très fréquenté (plusieurs centaines de connexions par jour).

Il se présente comme « un espace convivial entre internautes de toutes origines et qui partagent les valeurs universelles de tolérance, de civisme et de décence ». Il est réglementé par une charte dont le contenu correspond tout à fait à celui de la nétiquette dont il reconduit les grandes règles (voir supra) appelées dans ce forum « règles générales »:

« Il est interdit de poster un message à caractère publicitaire en faveur d'un site ou d'un produit ou d'un service. (...) Nous tenons à la règle "soyez poli" ; tout membre qui se montrerait intentionnellement désagréable ou perturbateur verra son compte suspendu sans préavis. »

Aux règles de politesse et de contenu des messages détaillées dans la charte, s'ajoute celle de la coopération également bien précisée, comme dans les extraits suivants :

Choisissez un titre approprié : le titre de votre sujet est très important. Choisissez des titres précis et clairs. (...). Soignez vos messages. Votre message doit traiter d'un sujet unique. Écrivez d'une manière claire et correcte et évitez le langage des SMS et Tchatche. (...) Soyez constructifs. Chaque topic traite d'un sujet particulier. Soyez constructifs et évitez les hors sujets. (...) Les sujets "fourre-tout" ne sont pas tolérés dans le forum.

a règle de coopération est présente aussi dans la consigne inhérente à la langue du forum. Son interface est en français mais le site reste ouvert aux autres langues parlées en Algérie comme l'arabe et le tamazight, sous certaines conditions :

La langue par défaut utilisée du forum est le français. Au cas où un membre est amené à citer un texte dans une autre langue, il est prié d'y ajouter la traduction en français s'il y a matière à débat (hors poèmes et paroles de chansons). L'écriture du style SMS n'est pas tolérée dans le forum vu qu'elle nuit à la lisibilité des messages.

Le respect de la nétiquette, dans ce forum, est quelque peu assuré par ses modérateurs qui reconnaissent s'en référer à l'autorégulation des messages par les internautes :

Bien que les administrateurs et les modérateurs du site algérie- $\mathrm{dz}$ essaient d'écarter tout message répréhensible de ce forum, il leur est impossible de contrôler tous les messages. Ceux-ci expriment uniquement les opinions de leurs auteurs. Les administrateurs et les modérateurs du forum n'entendent donner ni approbation, ni improbation des propos émis sur ce forum. Ils ne sauraient être considérés comme responsables du contenu des messages dont ils ne sont pas les auteurs.

Notre corpus est constitué des trente (30) messages du premier fil de la rubrique cuisine, intitulé «Farines utilisées en Algérie ». Il implique sept internautes à des degrés divers de participation. Signalons que nous avons reproduit tous les messages dans leur 
authenticité, c'est-à-dire tels qu'ils apparaissent sur le forum, sans procéder à aucune modification ou correction.

\section{Le style numérique du forum algérien au « niveau global»}

\section{Clarté et concision}

\section{Aghrroum g'imzen.}

Chez moi c'est Aghrum $\mathrm{n}$ temzin que je n'aime pas d'ailleurs, mais que tout le monde , surtout les vieux adorent. Thavarvucht $\mathrm{n}$ temzin aussi

Mister, je connais pas les noms en Français moi

je sais qu'il y' a timzin, irden pas. C'est un autre internaute, auteur du message $n^{\circ} 15$, qui le fera à travers un bref commentaire adressé à l'auteur du message, avant de revenir au sujet principal avec le partenaire intéressé par la réponse, MisterOver :

khouya Ahmed, Etouwham wech ydir...Loukane tqoulou lou2lou2 sidna soulayman ou ydjibha lha. Essaye le [la farine de Kamut] Sadiqi, tu ne vas pas le regretter. Mzebed ou m3assel naturellement. Il a un gout de noisette.

37 Ainsi la discussion sur l'épouse de MisterOver, c'est-à-dire sur sa vie privée, est close aussitôt qu'esquissée et celui qui l'a initiée disparaît de l'échange. 
C'est le cas également dans le message $\mathrm{N}^{\circ} 2$ : Absent commence en arabe par une considération générale sur le domaine de l'échange-»Djalassate etibakha moutawassila 3la hssab ma nchouf. $\|^{6}$ - aussitôt fermée par "Bon la différence entre... » qui marque à la fois la fin de la remarque en arabe et le retour au thème et à la langue française qui est celle de l'interface.

Dans les deux cas, la digression est mesurée, contrôlée soit par les autres internautes soit par son auteur.

La concision se lit également dans la progression des échanges, de leur ouverture à leur clôture. En effet, le message $\mathrm{N}^{\circ} 1$ inaugure la discussion :

Bonsoir les ami(e)s,

Tout à l'heure je suis passé dans un magasin bio et je me suis acheté plein de farines : seigle, sarasin, bis, semoule fin complet, semoule blanche de blé dur, ...

J'ai une question, quelles sont les noms de farine qu'on utilise en Algérie ? Comment on les appelait en arabe et en français?

En vous remerciant.

Et une question : C'est quoi la différence entre une farine de blé dur et une farine de blé tendre ? Est ce qu'on peut les utiliser de la même façon?

Les réponses d'auteurs différents se sont étalées sur une trentaine de messages jusqu'au dernier formulé sous forme de conclusion par un internaute qui a participé activement à l'échange mais qui n'est pas à l'origine des questions initiales :

41 Message $\mathrm{N}^{\circ} 30$

très intéressant le topic, merci MO.

tout a été dit ...

sauf peut être que les son de blé et d'avoine sont très bon pour la santé mais à consommer avec modération car irritent les intestins (pas plus de deux cuillère à soupe par jour je pense )

ps: j'adorais la galette au ch3ir quand j'étais petite, avec de l'huile d'olive, un déliceeeee

La formule "tout a été dit », même si l'internaute la restreint dans sa portée (« sauf ...»), signifie tout de même que la discussion a suivi un parcours logique, a évolué d'un début à une fin, dans le traitement du thème proposé.

42 Nous pouvons affirmer aussi que nous tenons là la preuve que le principe de coopération a été respecté, étant entendu que

Nos échanges de paroles ne se réduisent pas en temps normal à une suite de remarques décousues, et ne seraient pas rationnels si tel était le cas. Ils sont le résultat, jusqu'à un certain point au moins, d'efforts de coopération; et chaque participant reconnaît dans ces échanges (...) un but commun, ou un ensemble de buts, ou au moins une direction acceptée par tous. (Grice, op.cit. : 60)

43 De plus, ce respect de la cohérence et ce souci de la concision ne sont pas étrangers au ménagement des faces traité dans le point suivant.

\section{Ménagement des faces}

La totalité des messages révèle chez tous les participants le souci de la politesse et de la courtoisie, conformément aux recommandations des administrateurs du site. Les 
salutations avant d'introduire le message et pour le clore sont présentes dans presque tous les messages depuis le message $n^{\circ} 1$.

Selon Atifi (2003: 3),

La communication interpersonnelle médiatisée par l'Internet (...) met au premier plan la question du respect des règles du savoir communiquer. Ces règles sont explicitées dans divers textes constituant ce qu'on appelle la nétiquette - comme les chartes de forums. Elles font aussi partie des connaissances tacites dont disposent de nombreux internautes (...). Ainsi, de nombreuses formes d'échanges discursifs électroniques sont théoriquement réglementées par un contrat de communication préconisant certains comportements adéquats et prévoyant la sanction des comportements violant les règles.

Mais les formules de politesse les plus utilisées sont celles qui visent à installer une certaine convivialité entre participants comme dans les exemples suivants :

Message $\mathrm{N}^{\circ} 1:$ «Bonsoir les ami(e)s ... En vous remerciant »

Message $\mathrm{N}^{\circ} 2:$ : Bonsoir cousin, ... Wa choukrane.»

Message $\mathrm{N}^{\circ} 3$ : «Saha Sadiqi. Barak allahou fik cousin ...»

L'effet de cordialité que ces formules confèrent à l'échange est renforcé par de multiples remerciements mutuels pour ponctuer des évaluations positives des réponses les uns des autres :

Message $\mathrm{N}^{\circ} 17$ : « Exact Enoukhalla ! merci !...»

Message $\mathrm{N}^{\circ} 19$ : « Merci Sidinoun! oui c'est bien el khortel... »

Message $\mathrm{N}^{\circ} 22$ : «Merci beaucoup Megane, El Bahar, Sidi Noun... »

Message $\mathrm{N}^{\circ} 26$ : » @Sidi Noun : Merci beaucoup pour la traduction... »

Message $\mathrm{N}^{\circ} 28$ :» @El Bahar: Merci beaucoup Sadiqi, je suis en train de lire des trucs sur la farine du kamut, ça a l'air très intéressant ».

La nature bienveillante telle qu'affichée au cours de l'échange participe ainsi à la préoccupation de renvoyer une image positive de soi en ménageant celle de l'autre, l' ethos'de l'un se construisant dans sa compatibilité avec celui de l'autre.

Ce renvoi d'une image positive, ou " face » au sens de Goffman", se donne aussi à lire à travers d'autres actes de langage comme l'humilité qui transparaît de l'aveu d'ignorance de certains produits, et qui est une autre façon de solliciter l'autre, par exemple :

Message $\mathrm{N}^{\circ} 18:$ : ... en arabe je ne sais plus ... Khortal...... »

Message $\mathrm{N}^{\circ} 19$ : » ... Pour l'épeautre je ne sais pas si ca existe chez nous MisterOver...... » Message $\mathrm{N}^{\circ} 21$; »... Il y a des céréales comme le seigle, le sarrasin, l'épeautre dont je n'ai jamais entendu parler en Algérie, je ne sais pas si ca existe la bas... »

Message $\mathrm{N}^{\circ} 25:$ «... Le tilleul, à ma connaissance, n'est pas utilisé en Algérie...... »

Mais ce genre d'aveu prend place dans un contexte de préservation explicite de la face en mobilisant plusieurs stratégies.

51 L'internaute invoque un contexte social (et/ou historique) qui ne lui permet pas d'identifier un produit :

Message $\mathrm{N}^{\circ} 4:$ «... Sinon chez moi d'au moins toutes el kesrate en semoule donc en blé dur et tous les gâteaux (ou presque) avec de la farine du blé tendre.

Fi waqti ana, il n'y avait qu'une seule farine : la farine au blé tendre. La panifiable. (D'au moins elles étaient toutes vendues comme étant des farines panifiables).» 
Message $N^{\circ} 7:$ «Aghrroum g'imzen. Chez moi c'est Aghrum n temzin que je n'aime pas d'ailleurs, mais que tout le monde, surtout les vieux adorent . Thavarvucht $n$ temzin aussi

Mister , je connais pas les noms en Français moi

je sais qu'il y' a timzin, irden. »

L'internaute promet de combler ses lacunes pour rendre service à son interlocuteur :

Message $\mathrm{N}^{\circ} 18:$ « ... Le son de blé (j’ai oublié comment on appelle ça en arabe...si ça me revient je le rajouterai) en kabyle c'est agouchel... »

Cette attitude de coopération, qui s'inscrit dans la catégorie "qualité » du principe de coopération de Grice, participe aussi de la convivialité. Celle-ci est, selon Marcoccia, l'une des règles de base de la nétiquette au niveau global (voir supra).

Contribue aussi à ce ménagement de la face, la construction de l'identification des internautes. En effet, ils recourent tous aux pseudonymes, procédé courant chez les internautes qui ne déclinent quasiment jamais leur identité : MisterOver, Absent, Tite-fée, Megane, Sidi Noun, ahmed67, absente.

Ce choix participe de la face en ce qu'il est librement choisi pour s'auto-désigner, contrairement au sobriquet

De plus, leur caractère arbitraire est accentué par l'impossibilité de leur associer même partiellement un sens dans le monde réel; même si dans le cas de Megane et de Sidi Noun, on ne peut s'empêcher de penser à la marque de véhicule pour le premier et à la mosquée de Tlemcen pour le second.

Cette valorisation est renforcée par le recours à l'anglais et au français dans la quasitotalité des cas.

Les seuls pseudonymes qui échappent à ce caractère arbitraire par leur motivation supposée sont les suivants : «absente », féminin, qui semble dicté par sa contiguïté avec «Absent», masculin, et par son absence dans le fil à l'exception d'une seule fois, en dernière position ; pour le second, « ahmed67 », le chiffre 67 accolé au prénom Ahmed a été choisi par l'internaute pour une raison précise même si celle-ci n'est pas dévoilée dans l'échange.

De plus, un certain nombre d'indices, distillés au cours de la discussion, permet une identification de l'internaute au niveau socioculturel, relativisant fortement l'anonymat affiché. Prenons l'exemple des deux forumeurs principaux, Absent et MisterOver, à travers quelques extraits de leurs messages :

«Absent »:

Message $\mathrm{N}^{\circ} 4$

Sinon chez moi d'au moins toutes el kesrate en semoule donc en blé dur et tous les gâteaux (ou presque) avec de la farine du blé tendre.

Fi waqti ana, il n'y avait qu'une seule farine : la farine au blé tendre. La panifiable

Message $\mathrm{N}^{\circ} 6$

Donc la farine de l'orge? Aghrroum g'imzen.

Message $\mathrm{N}^{\circ} 8$

Exact! C'est Aghroum n' thimzine. Khoubz ec3ir. Pain d'orge.

Et Aghroum n' ggirdhen pour pain avec semoule de blé.

J'ai perdu mon kabyle 


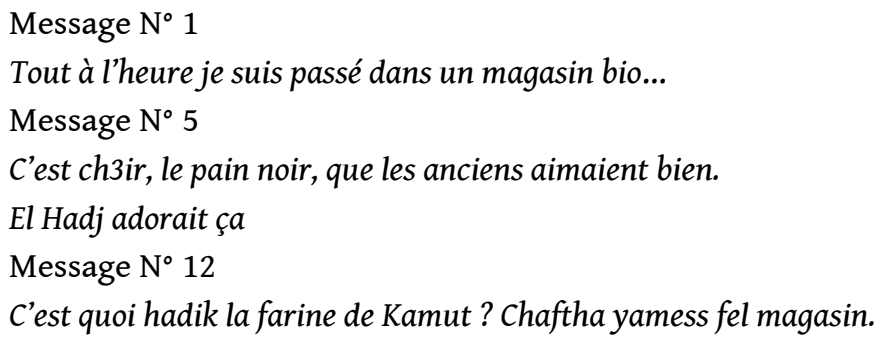

Mais une question: El Noukhala, khartal, on le donnait aux bêtes chez Nous. Je me souviens, c'était moi qui mélangeais el Noukhala, je mettais de l'eau avec, ils l'adoraient les brebis.(..) Honnêtement, je trouve ça drôle, cette chimie, ce mélange. (...) Disons qu'il me faut toujours une petite passion, avant c'était la littérature, histoire, politique, cinéma, ..... mais depuis qq années tout ça m'a saoulé, je reviens aux sources, j'avais commencé par : Les huiles essentielles, plantes \& tisanes, huiles végétales, et après je me suis lancé dans la cuisine, et dernièrement pâtisserie. Mais je reste débutant, mais cela dit, je trouve ça excellent, beaucoup plus interessant que l'Informatique, Math, ... la science des aliments, les plantes, les huiles, ....

Message $\mathrm{N}^{\circ} 26$

En plus, il y'a plein de nanas qui le cherchent (l'avoine) sur les forums de santé, et tout pour le régime Dukan (...).En regardant chouia sur le Net, apparement la Tillel c'est zayzafoune, mais je n'ai jamais entendu parler de ça chez Nous.

MisterOver est donc un Algérien, qui a vécu à la campagne, relativement jeune eu égard à certaines de ses expressions ("plein de nanasqui...»), vit à l'étranger et vraisemblablement en France (cf. message $n^{\circ} 1$ : le produit est étiqueté en français), a des centres d'intérêt très diversifiés et exclusifs (" avant c'était ...mais depuis qq années ... je reviens, j'avais commencé...et après je me suis lancé...et dernièrement pâtisserie... »). En un mot c'est un émigré, instruit, passionné, moderne au sens où il s'inscrit dans la tendance nouvelle de la société du pays où il réside (hygiène alimentaire "bio ", engouement pour la cuisine, au courant des régimes amincissants,...).

Ainsi, les messages sont certes anonymes, mais distillent des renseignements sur le profil de leurs auteurs. L'effet d'une telle stratégie est double : contribuer à la fois au ménagement de la face et au respect de règle d'identification de la nétiquette de ce forum algérien de discussion.

C'est également le cas des smileys.

\section{Le smiley ou subjectivème}

61 Pour achever de montrer que le forum algérien respecte les règles standard du genre, nous avons réservé un dernier point aux smileys, ou emoticons, qui sont selon Marcoccia (2000):»combinaisons de topogrammes et de caractères d'imprimerie permettant de 
représenter de manière schématique (...) des mimiques faciales comme des sourires (...), des clins d'œil (...), des moues de colère ou de tristesse (...).

\section{Message $\mathrm{N}^{\circ} 2$}

Djalassate etibakha moutawassila 3la hssab ma nchouf. $\$$ Bon la différence entre..

Message $\mathrm{N}^{\circ} 22$

Ah oui, je comprends mieux, c'est pour ça que le son de blé ressemble à Noukhala Wण

Message $\mathrm{N}^{\circ} 3$

Barak allahou fik cousin, très bonne explication.

Message $\mathrm{N}^{\circ} 5$

El Hadj adorait ça, surtout l'hivers avec du beurre et Lben $\theta$

Message $\mathrm{N}^{\circ} 26$

@Megane: En plus, il y'a plein de nanas qui le cherchent (l'avoine) sur les forums de santé, et tout pour le régime Dukan, j'aurais pu les envoyer chez mon oncle, il a plein de Khourtal $\Theta$

Message $\mathrm{N}^{\circ} 7$

Mister , je connais pas les noms en Français moi

Message $\mathrm{N}^{\circ} 30$

(...) à consommer avec modération car irritent les intestins (pas plus de deux cuillère à soupe par jour je pense)

ps :j'adorais la galette au ch3ir quand j'etais petite, avec de l'huile d'olive, un déliceeeee

Nous remarquons que leur fonction dans ce forum est essentiellement expressive mais non redondante par rapport au contenu du message. Les smileys de notre corpus n'illustrent pas ce qui a déjà été dit dans le texte, mais ajoutent de l'information au texte en révélant la disposition de l'auteur vis-à-vis de ce qu'il vient d'écrire. l'énonciation de l'énoncé. Ils fonctionnent donc comme des indices de l'énonciation un internaute, à travers Zen (smiley animé : baisse et relève la tête), reçoit l'explication 
attendue avec sérénité/satisfaction et reconnaissance (message $\mathrm{N}^{\circ} 3$ ); un autre, à travers Mrgreen, est content que les discussions autour de la cuisine continuent (message $\mathrm{N}^{\circ} 2$ ) ; un autre encore, à travers la duplication de Mrgreen, est doublement content quand il découvre (au cours de la discussion) que le « son de blé » ressemblait à « Noukhala » (message $\left.\mathrm{N}^{\circ} 22\right)$; MisterOver ponctue par BigGrin/grand sourire l'évocation de son père (message $\mathrm{N}^{\circ} 5$ ) ou, pour plaisanter, sa proposition d'envoyer les «nanas " qui recherchent l'avoine recommandée dans le « régime dukan » chez son oncle parce qu'il vient de comprendre que c'est le khourtal qu'on donnait aux bêtes chez lui (en Algérie); Megane exprime par le biais de confused (qui fait la grimace) son désappointement de ne pas savoir les noms des céréales demandés en français ; absente enfin parachève son message neutre sur les effets indésirables d'une consommation excessive de son de blé sur les intestins, par razz ou la moquerie; elle fait de même avec redface/timidité pour tempérer l'ardeur dont elle a investi typographiquement son message pour signifier l'intensité de son sentiment pour la galette de son enfance.

Donc, grâce aux smileys, que l'internaute charge (voire suresvestit) de ses sentiments à défaut de les verbaliser, les messages gagnent en émotion sans pour autant déroger à la règle de la nétiquette qui interdit les messages trop personnels. Dans le cas de ce forum, les smileys, tout en étant cadrés par la nétiquette, sont une véritable valeur ajoutée au niveau de la relation de communication.

68 Au regard des points étudiés jusqu'ici, nous pouvons donc affirmer que ce forum s'est déroulé dans le strict respect des règles de standardisation.

Cependant, il reste un aspect des échanges qui n'a pas été pris en charge au cours de cette tentative d'analyse du style numérique au niveau global parce qu'il relève du style numérique au niveau local. A défaut d'en faire une démonstration exhaustive, nous allons en pointer quelques données qui nous semblent pertinentes.

\section{Le style numérique du forum algérien au « niveau local » : la stratégie de l'alternance codique}

69 Notre objectif est d'appréhender les modalités spécifiques de la présence de quelques traits propres à la culture des internautes dans ce forum francophone, pour étayer, à notre niveau la thèse du double style des forums telle que prônée par M. Marcoccia et H. Atifi.

70 Le premier type de présence culturelle des internautes est la thématisation de la culture algérienne, par le biais d'évocations, par les internautes, d'habitus dans les trois langues : français, arabe et kabyle.

71 Cette description est sollicitée par le topic du forum motivé non par la reconnaissance d'une altérité "(chez nous" vs chez eux), mais plutôt comme une tentative de décodage de ce qui existe dans le «magasin bio »/» (ici, en France, pour MisterOver, la bas pour Megane), et qu'il ne sait pas identifier par rapport à la connaissance acquise « en Algérie » (la bas pour MisterOver).

72 Chaque internaute dans sa contribution au projet de réponse va parler de ce qu'il sait ou ne sait pas des "farines" de chez lui, en Algérie; d'où le recours pléthorique à la locution de localisation "en Algérie ", modalisée à travers ses déclinaisons en "chez moi »/ » chez nous »/ " en Kabylie »/ » la bas »/ etc., ; d'où également la forte présence du présentatif "c'est» suivi de nombreuses identifications ou de questions qui les 
sollicitent: "c'est agourchel», "c'est bien el khortel», "C'est ch3ir», "C'est quoi la différence », etc.

Mais la présence thématique, descriptive, de la culture algérienne, est une sorte de degré zéro de la spécificité culturelle du forum parce qu'elle ne détermine pas, en tant que telle, la structuration intrinsèque des messages. En d'autres termes, cette présence en tant que thème n'influe pas sur «les normes du savoir communiquer " (Atifi, 2003, op.cit.).

Or, notre objectif est d'essayer de cerner la modalité à travers laquelle la spécificité culturelle en situation de communication imprime sa trace dans la matérialité des messages échangés, pourtant cadrés par la nétiquette.

Nous allons en faire une brève démonstration en étudiant quelques manifestations de l'alternance codique au niveau des appellatifs/ouvertures du forum.

Reprenons les messages dans l'ordre de leur apparition sur le forum :

$\mathrm{N}^{\circ} 1$ : MisterOver : «Bonsoir les ami $(e)$ s »

$\mathrm{N}^{\circ} 2$ : Absent : «Bonsoir cousin»

$\mathrm{N}^{\circ} 3$ : MisterOver : «Saha Sadiki Barak allahou fik cousin »

$\mathrm{N}^{\circ} 7$ : Tite-fée : «Mister, je connais pas les noms en Français moi »

$\mathrm{N}^{\circ} 11$ : Absent : «Saha Sadiqi»

$\mathrm{N}^{\circ} 12$ : MisterOver : « Saha sadiqi El bahar »

$\mathrm{N}^{\circ} 13:$ ahmed67 : « mister over tu cherche a te recycler »

$\mathrm{N}^{\circ} 14$ : Megane : «Bonsoir MisterOver »

$\mathrm{N}^{\circ} 15$ : Absent : «khouya Ahmed »- «Essaye le Sadiqi»

$\mathrm{N}^{\circ} 19$ : Megane : «Merci Sidinoun»- «Pour l'épeautre je ne sais pas si ca existe chez nous MisterOver"

$\mathrm{N}^{\circ} 20$ : Absent : «De rien Megane »

$\mathrm{N}^{\circ} 21$ : Megane : «El Bahar j'ai entendu aussi echoufane »

№ 22: Mister Over: «Merci beaucoup Megane, El Bahar, Sidi Noun»- «@Ahmed:

Honnêtement... »

N²6: MisterOver : «@Megane : En plus... »- «@Sidi Noun : Merci beaucoup... »

$\mathrm{N}^{\circ} 28$ : MisterOver : « @El Bahar : Merci beaucoup Sadiqi... »

$\mathrm{N}^{\circ} 29$ : Absent : « Heqqa, Etemina...ana sadiqek yak? Ma tensaniche hein?»

$\mathrm{N}^{\circ} 30$ : absente : « très interessant le topic, merci $M O$ ».

77 Nous remarquons d'emblée que l'alternance codique installe un code hiérarchisé des relations entre les forumeurs. La mesure est donnée par les salutations/interpellations entre MisterOver et Absent.

Dans les trois messages successifs qu'ils ont échangés au début du topic ( $\mathrm{N}^{\circ} \mathrm{s}_{1}, 2$ et 3$)$, au nom générique en français « ami(e) $s$ » pour un salut qui ne préjuge pas de relation d'amitié mais simplement de cordialité, répond d'abord le nom en français de parenté masculine, " cousin ", mais dans le sens utilisé en Algérie pour signifier un salut cordial dans le même sens qu'« ami ».

Donc, la superposition de "bonsoir les ami(e)s » et de "bonsoir cousin», autorise la permutation de « $\operatorname{ami}(\mathrm{e}) \mathrm{s}$ »/ami et « cousin » et par conséquent leur équivalence. Nous en déduisons que, dans le contexte algérien du forum, c'est-à-dire dans celui d'une communication interactive, "ami» pour saluer un vis-à-vis masculin, se dit aussi « cousin » (nous verrons ce qu'il en est du féminin). 
Le message $\mathrm{N}^{\circ} 3$ apporte une nuance sémantique supplémentaire par la contiguïté de " sadiki » et de « cousin ». Par cette juxtaposition, " cousin », équivalent d' " ami » en français, n'est pas celui de « sadiki » qui pourtant signifie « mon ami » en arabe.

81 Et dans la suite de l'échange entre Mister Over et Absent, "ami » et "cousin » sont évincés puisqu'ils ne s'interpelleront plus qu'à travers ce mot arabe «sadiki»: messages $11,12,15,28$ et 29 .

« Saha sadiki » se substitue à «bonsoir les ami(e)s »/ » bonsoir cousin »; "Sadiki » n'est ainsi ni « ami » au sens générique du mot français, ni son équivalent en français de « cousin » dans le contexte algérien, ni l'équivalent du second pseudonyme d'Absent, dans le message $\mathrm{N}^{\circ} 28$ (« @El Bahar : Merci beaucoup Sadiqi... »).

MisterOver distingue très nettement l'identification ( $E l$ Bahar/Absent en tant que destinataire du message) de l'appellatif (Sadiki). en arabe parce que le mot présuppose une relation non pas de cordialité (comme en français) mais d'amitié. La preuve en est que dans le dernier message d'Absent à MisterOver, cette relation d'amitié est posée, thématisée: "ana sadiqek yak? Ma tensaniche hein?» [moi, je suis ton ami, n'est-ce pas? ne m'oublies pas, hein ?]. Cette relation d'amitié qui ne se décline qu'en arabe est d'autant plus privilégiée qu'avec les autres forumeurs, l'échange reste protocolaire, aussi bien avec les filles qu'avec les garçons mais peut-être pas pour les mêmes raisons :

MisterOver appelle tous les autres partenaires (filles ou garçons) par leurs pseudonymes ;

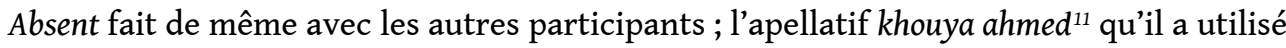
une fois (message $\mathrm{N}^{\circ} 15$ ) en s'adressant à ahmed67 n'est pas le signe d'un rapprochement fraternel puisque dans le même message, il est distingué de Sadiki dont il use quand il s'adresse à MisterOver dans le même message; pour Absent, " khouya ahmed » n'est pas synonyme de « Sadiki »;

Il en est de même des autres internautes qui recourent également aux pseudonymes ; Chez les filles, le procédé peut aller jusqu'à la déférence (cf. Mister ${ }^{12}$ : message $\mathrm{N}^{\circ} 7$ ) ; Sidi Noun n'use ni d'appellatif, ni autre formule d'ouverture ou de fermeture du message.

Fig. 1 : tableau récapitulatif des appellatifs du fil

\begin{tabular}{|l|l|l|l|l|l|l|l|}
\hline Appellatifs & MisterOver & $\begin{array}{l}\text { Absent / El } \\
\text { Bahar }\end{array}$ & Megane & Ahmed67 & $\begin{array}{l}\text { Tite- } \\
\text { fée }\end{array}$ & $\begin{array}{l}\text { Sidi } \\
\text { Noun }\end{array}$ & absente \\
\hline pseudonyme & $\mathrm{x}$ & $\mathrm{x}$ & $\mathrm{x}$ & $\mathrm{x}$ & $\mathrm{x}$ & $\mathrm{x}$ & $\mathrm{x}$ \\
\hline « ami(e)s» & & $\mathrm{x}$ & $\mathrm{x}$ & $\mathrm{x}$ & $\mathrm{x}$ & $\mathrm{x}$ & $\mathrm{x}$ \\
\hline « Mister» & $\mathrm{x}$ & & & & & & \\
\hline « cousin» & $\mathrm{x}$ & $\mathrm{x}$ & & & & & \\
\hline « sadiki » & $\mathrm{x}$ & $\mathrm{x}$ & & & & & \\
\hline « khouya ahmed & & & & $\mathrm{x}$ & & & \\
\hline
\end{tabular}


86

\section{BIBLIOGRAPHIE}

AMOSSY R., La Présentation de soi : Ethos et identité verbale, P.U.F., Paris, 2010,

ANIS J., " Dispositifs multimédias et conversation électronique : approche sémiolinguistique », in

Cahiers du français contemporain, $\mathrm{n}^{\circ} 6$, Multimédia : les mutations du texte, Lancien $\mathrm{T}$. (dir.), ENS, Fontenay St-Cloud, 2000, pp. 57-86.

ATIFI H., «Variation culturelle dans les communications en ligne : analyse ethnographique des forums de discussion marocains ", in Langage et société, $\mathrm{N}^{\circ} 102$, Maison des sciences de l'homme, 2003/2, pp. 57-82. Disponible sur le site :

http://www.cairn.info/revue-langage-et-societe-2003-2-page-57.htm.

ATIFI H. ET MARCOCCIA M., « Communication médiatisée par ordinateur et variation culturelle : analyse contrastive de forums de discussion français et marocains ", in Les Carnets du Cediscor [En ligne], 9 | 2006. Mis en ligne le 01 avril 2008. Disponible sur le site : https:// cediscor.revues.org/629

CAUBET D., « Métissages linguistiques ici (en France) et là-bas (au Maghreb) », in Ville-EcoleIntégration Enjeux : pratiques langagières urbaines. Enjeux identitaires, enjeux cognitifs, $\mathrm{n}^{\circ} 130$, septembre, CNDP, Paris, 2002, pp. 117-132.

DABENE L., Repères sociolinguistiques pour l'enseignement des langues, Hachette, Paris, 1994. KERBRAT- ORECCHIONI C., Les interactions verbales, Tome 1, Armand Colin, Paris,1990.

GoffMAn E., La Mise en scène de la vie quotidienne, Tome 1, La Présentation de soi, Minuit, Paris, 1973.

Multilinguales, 7 | 2016 
GOFFMAN E., Les rites d'interaction, Minuit, Paris, 1974.

GRICE H.P., "The logic and conversation, syntax and semantics", dans vol. III, Speech Acts, ed. Par P. Cole et J.L. Morgan, Academic Press, Inc., 1975, p. 41-58. Traduit de l'américain par F.Berthet et M. Bozon : «Logique et conversation », in Communication, $\mathrm{N}^{\circ}$ 1, La conversation, volume 30, 1979, pp. 57- 72. Traduction disponible sur le site : http://www.persee.fr/doc/ comm_0588-8018_1979_num_30_1_1446.

HAMBRIDGE S., Netiquette Guidelines, McGraw-Hill, New York, 1995.

MAINGUENEAU D., Discours et analyse du discours, Armand Colin, Paris, 2014.

MANGENOT F., « Du Minitel aux SMS, la communication électronique et ses usages pédagogiques », Linx [En ligne], 60 | 2009. Mis en ligne le 28 mars 2012. Consulté sur le site https://linx.revues.org/702

MARCOCCIA M., « La normalisation des comportements communicatifs sur Internet : Etude sociopragmatique de la netiquette ", dans Guerin N. \& Toblin L., (Ed), Communication, Société et Internet, L'Harmattan, Paris, 1998, pp. 15-22.

MARCOCCIA M., « La politesse dans les forums de discussion sur Internet. Règles externes, manifestations discursives et commentaires métacommunicatifs », dans Dialogue Analysis 2000 Selected Papers from the 10th Iada Anniversary Conference, Bologna 2000 (Beitrage zur Dialogforschung), Marina Bondi and Sorin Stati, (Ed.), EditeurTübingen, M. Niemeyer Verlag, 2003, pp. 315-326.

MARCOCCIA M., «L'analyse conversationnelle des forums de discussion », in Les discours de l'internet : nouveaux corpus, nouveaux modèles ?, Les carnets du Cediscor8, Presses Sorbonne nouvelle. 2004, pp. 23-38.

MARCOCCIA M., GAUDUCHEAU N. «L'analyse du rôle des smileys en production et en réception : un retour sur la question de l'oralité des écrits numériques ", in Glottopol, N 10, 2007, pp. 38-55.

PANCKHURST R., « Short Message Service (SMS) : typologie et problématiques futures ", in Polyphonies, pour Michelle Lanvin, Arnavielle T., (coord.), Université Paul-Valéry Montpellier 3, 2009, pp. 33-52.

PANCKHURST R., «Le discours électronique médié : bilan et perspectives », dans Lire, écrire, communiquer et apprendre avec Internet, A. Piolat, (dir), Éditions Solal, Marseille, 2006, pp. 345-366.

RINALDI A., The Net: User Guidelines and Netiquette, Florida Atlantic University,1996. Disponible sur le site : ourses.cs.vt.edu/ cs3604/lib/Netiquette/Rinaldi/.

SHEA V., Netiquette, , Albion Books, San Francisco CA, 1994.

\section{NOTES}

1. J. Anis à titre indicatif a notamment publié :

"Ordinateur, pédagogie, communication", in Langue française, $\mathrm{n}^{\circ} 70$, Communication et enseignement, M. Verdelhan-Bourgade (dir.), Larousse, Paris, 1986, pp. 98-111.

Texte et Ordinateur. Les Mutations du lire-écrire, LINX hors série, Actes du colloque interdisciplinaire tenu à l'Université de Nanterre, 6-8 juin 1990, J.Anis et J.-L. Lebrave (dirs.), Université de Paris XNanterre, 1990. 
«Approche sémiolinguistique d'interactions écrites en temps réel sur l'Internet », in R. Bouchard \& F. Mangenot (eds.), Interactivité, interactions et multimédia, coll. « Notions en question », $\mathrm{n}^{\circ} 5$, ENS éditions, Fontenay Saint-Cloud, 2001, pp. 147-188.

Parlez-vous texto? Guide des nouveaux langages du réseau, J. Anis (dir.), Paris, Le Cherche-Midi, 2001. "Communication électronique scripturale et formes langagières: chats et SMS ", Actes des Quatrièmes Rencontres Technologiques, 31 mai-1 juin 2002, 2003, Université de Poitiers.

2. A ce stade de nos recherches, nos observations et conclusions ne peuvent être généralisées à tous les forums algériens.

3. M. Marcoccia avait déjà traité ce sujet deux ans auparavant dans un article intitulé «La normalisation des comportements communicatifs sur Internet : étude sociopragmatique de la Nétiquette", dans N. Guégen et L. Tobin (eds.), Communication, société et Internet, Paris, L'Harmattan, 1998, pp. 15-32.

4. H. P. Grice expose sa théorie du principe de coopération, en 1975, dans l'article « Logic and conversation ", traduit de l'américain en 1979 : «Logique et conversation », in Communication, $\mathrm{N}^{\circ} 1$, volume 30, La conversation, 1979, pp.57- 72. Ce principe se compose de quatre catégories traduites en : "Quantité » (informer mais de façon nécessaire et suffisante) ; "Qualité » (n'informer que de ce que l'on croit vrai ou vérifiable); « Relation» (interventions appropriées); «Modalité» (être clair, concis et bref dans ses interventions)» (cf. pp. 61-62). L'article traduit est consultable sur le site :

< http://www.persee.fr/doc/comm_0588-8018_1979_num_30_1_1446>.

5. Le lien du forum :

<algérie-dz.com www.algerie-dz.com/forums/archive/index.php>

6. Traduction libre : [les discussions ( ?) autour de la cuisine continuent à ce que je vois].

7. Selon Ruth Amossy, «il ne s'agit pas de ce que le sujet est [...] mais de l'image qu'il projette dans une situation précise » $(2010: 27)$.

8. Pour Goffman E., la face est « la valeur sociale positive qu'une personne revendique effectivement à travers la ligne d'action que les autres supposent qu'elle a adoptée au cours d'un contact particulier» (1974: 9).

9. «Fi waqti ana " [de mon temps].

10. Ils correspondent au subjectivème que Catherine Kerbrat-Orecchioni définit comme " énonciateur qui s'avoue explicitement ou se pose implicitement comme la source évaluative de l'assertion » (1990: 80).

11. [Mon frère] ou [mon frère + prénom] : appellatifs très usités en Algérie, pour s'adresser poliment à quelqu'un du genre masculin sans aucune implication de lien de parenté.

12. [Monsieur].

\section{RÉSUMÉS}

Cette étude se focalise sur le forum de discussion algérie-dz.com, en tant que nouveau genre discursif, dans sa double dimension : "globale» (internationale) et «locale» (nationale). Nous avons d'abord dressé l'état de l'art des forums. Nous nous sommes attelé ensuite à l'étude de ce type de pratiques discursives. Leur analyse détaillée nous a permis de cerner les procédés dont usent les internautes pour mettre en œuvre des stratégies de communication, des formules 
d'adresse et d'interpellation, des registres de langue, dont certains relèvent du style standard de la « nétiquette » et d'autres du style de la culture locale.

This study focuses on the discussion forum Algérie-DZ.com as a new discursive genre, in its double dimension: "global" (international) and "local" (national). We have first outlined the state of the art of the forums. Then, we got interested in the study of this type of discursive practices. Their detailed analysis allows us to understand the processes adopted by internet users to establish communication strategies, addressing and challenging formulas, language registers, some of them fall within the standard style of "netiquette" and others of the local culture style.

\section{INDEX}

Mots-clés : Principe de coopération, forum de discussion, alternance codique, interaction, nétiquette, smiley, style numérique, algérie-dz.com

Keywords : Coorporation principe, discussion forum, coding alternance, interaction, netiquette, digital style, algérie-dz.com

مبدأ التعاون, منتدى مناقشات, تناوب شفري, تفاعل, آداب شبكة فهرس الكلمات المفتاحية: الإنترنت (نيتيكات), سم أسلوب رقمي

\section{AUTEUR}

\section{YAHIA ABDELDJEBAR ATMANE}

Université Djilali Liabès- Sidi-Bel-Abbès - Algérie 